University of Nebraska - Lincoln

DigitalCommons@University of Nebraska - Lincoln

Uniformed Services University of the Health

Sciences

U.S. Department of Defense

2011

\title{
Comparison of Combat Gauze and TraumaStat in Two Severe Groin Injury Models
}

\author{
Françoise Arnaud \\ Naval Medical Research Center, francoise.Arnaud@med.navy.mil \\ Kohsuke Teranishi \\ Naval Medical Research Center \\ Tomoaki Okada \\ Naval Medical Research Center \\ Dione Parreño-Sacdalan \\ Uniformed Services University of Health Science \\ Daniel Hupalo \\ Naval Medical Research Center \\ See next page for additional authors
}

Follow this and additional works at: https://digitalcommons.unl.edu/usuhs

Part of the Medicine and Health Sciences Commons

Arnaud, Françoise; Teranishi, Kohsuke; Okada, Tomoaki; Parreño-Sacdalan, Dione; Hupalo, Daniel; McNamee, George; Carr, Walter; Burris, David; and McCarron, Richard, "Comparison of Combat Gauze and TraumaStat in Two Severe Groin Injury Models" (2011). Uniformed Services University of the Health Sciences. 69.

https://digitalcommons.unl.edu/usuhs/69

This Article is brought to you for free and open access by the U.S. Department of Defense at DigitalCommons@University of Nebraska - Lincoln. It has been accepted for inclusion in Uniformed Services University of the Health Sciences by an authorized administrator of DigitalCommons@University of Nebraska Lincoln. 


\section{Authors}

Françoise Arnaud, Kohsuke Teranishi, Tomoaki Okada, Dione Parreño-Sacdalan, Daniel Hupalo, George McNamee, Walter Carr, David Burris, and Richard McCarron 


\title{
Comparison of Combat Gauze and TraumaStat in Two Severe Groin Injury Models
}

\author{
Françoise Arnaud, Ph.D., ${ }^{*}+, 1$ Kohsuke Teranishi, M.D., ${ }^{*}$ Tomoaki Okada, M.D., ${ }^{*}$ \\ Dione Parreño-Sacdalan, M.D.,† Daniel Hupalo, M.S., ${ }^{*}$ George McNamee, D.M.V.,† Walter Carr, Ph.D., \\ David Burris, M.D., $\dagger$ and Richard McCarron, Ph.D.*, $\dagger$ \\ *Department of Trauma and Resuscitative Medicine, Naval Medical Research Center, Silver Spring, Maryland; and $\dagger$ Department of \\ Surgery, Uniformed Services University of Health Science, Bethesda, Maryland \\ Submitted for publication July 7, 2009
}

Background. Fabric-like hemostatic dressings offer promise for hemorrhage control in noncompressible areas, especially given their similarity in form to standard gauze currently in use. Recently, two such products, Combat Gauze (CBG) and TraumaStat (TMS), were introduced. Their performance is evaluated in two vascular injury models.

Materials and Methods. The dressings were evaluated in anesthetized Yorkshire pigs, hemorrhaged by full transection of the femoral vasculature with 2 min free bleeding period $(\mathrm{CBG}=6$, TMS $=6$ ) or by $4 \mathrm{~mm}$ femoral arterial puncture with 45 s free bleeding period $(C B G=8, T M S=8)$. After injury, dressings were applied, followed by 5 min of manual compression and then $500 \mathrm{~mL}$ resuscitation fluid infused over 30 min. Vital signs, blood pressure, and blood loss were recorded throughout the 3 -h experiment. Bleeding control was the primary outcome.

Results. All animals had similar pretreatment mean arterial pressure (MAP) ( $36.5 \mathrm{mmHg})$; pretreatment blood loss following injury was similar for both dressing groups in the two models [24\% $\pm 8 \%$ estimated blood volume (EBV) 2 min after transection and $17 \% \pm 4 \%$ EBV $45 \mathrm{~s}$ after puncture. Incidence of post-treatment bleeding, primarily occurring after release of manual compression or restoration of blood pressure, was more frequent in the puncture model (17\% with both CBG and TMS) than the transection model (57\% with CBG versus 75\% with TMS). Post-treatment blood loss not controlled by the dressing was $19 \% \pm 22 \%$ and $31 \% \pm 17 \% \mathrm{EBV}$, for CBG and TMS, respectively. Survival rate was $100 \%$

${ }^{1}$ To whom correspondence and reprint requests should be addressed at Naval Medical Research Center, RMD, Trauma and Resuscitative Medicine, 503 Robert Grant Avenue, Silver Spring, MD 20910-7500. E-mail: francoise.Arnaud@med.navy.mil. for both dressings in the transection model, and was 88\% for CBG and $50 \%$ for TMS in the puncture model.

Conclusions. These findings indicated that CBG and TMS were similarly effective in improving hemostasis. These two fabric-like dressings showed easy application and removal, leaving a clean wound for surgical repair. ๑ 2011 Elsevier Inc. All rights reserved.

Key Words: hemorrhage; bleeding control; hemostatic dressings; bandages; trauma; hemostasis; swine models.

\section{INTRODUCTION}

Mortality from hemorrhagic shock caused by massive bleeding in a wound is preventable if acted upon immediately after injury. With proper compression on the wound site, use of adequate hemostatic dressing for bleeding control, timely fluid resuscitation for hemodynamic restoration, and definitive management, complications such as compartment syndrome, limb loss, or death can be minimized. In an effort for better hemostatic control in noncompressible areas such as the neck or the groin where tourniquets cannot be used, a variety of highly effective hemostatic dressings have become available and can be selected according to various qualities like their physical nature. In a previous series of experiments comparing 10 hemostatic dressing products to compressed gauze (as the standard of care) $[1,2]$, three products, X-Sponge (Z-Medica, Wallingford, CT), a synthetic gauze coated with kaolin; Woundstat (TraumaCure, Bethesda, MD), a smectite/polymer granular product; and Celox (Sam Medical Products, Newport, OR), a chitosan-based product, were found to be the most effective dressings for survival, blood loss, 
and bleeding control among those tested. A key observation in those experiments was that the fabric-based products (e.g., X-Sponge) were similar in form to standard gauze and could be relatively easily removed, leaving a clean wound, ready for surgical repair.

After completion of our previous study, two products, (1) Combat Gauze (CBG; Z-Medica, Wallingford, CT), and (2) TraumaStat (TMS; Ore-Medix, Salem, OR), were newly introduced and proposed for experimental comparison. CBG was essentially a next generation of $\mathrm{X}$-Sponge but configured as a roll of kaolin-coated polyester gauze rather than the $4 \mathrm{in}$. by $4 \mathrm{in}$. gauze format of $\mathrm{X}$-Sponge. TMS was a chitosan-based chemical formulation, a similar agent as used in Celox, but TMS was supported on a solid matrix rather than presented as a loose powder as Celox. These two new dressings are compact fabric-like dressings, a quality that is similar to the current standard gauze, and, based on our experience with previous hemorrhage control experimentation, this supports easy application and removal from the wound. This type of dressing is well suited for rapid deployment in the battlefield [3-5].

It is recognized that massive bleeding approaching $45 \%$ of original blood volume is devastating, particularly if there is a secondary bleeding (rebleeding) during the compensation phase $[4,5]$. Two injury models [6-13], a full femoral transection (including artery and vein) and a femoral artery puncture yield massive blood loss in the early phase of bleeding and severe hemorrhagic shock consequences if untreated. Our previous results indicated that dressing efficacy outcomes were different across these two injury models $[1,2]$. There was more post-treatment bleeding after the compensatory phase in the puncture injury model than the transection. Therefore, the use of two models provides a broader span of challenge to test dressings. It is an important consideration while testing hemostatic dressings in situations where there are high risks of excessive blood loss.

We report on the evaluation of two relatively new fabric-like hemostatic dressings in these two surgical models, developed to simulate injuries from the battlefield $[1,2]$ and to address various levels of vascular injury. The objective of this study is to evaluate the efficacy of CBG and TMS. The specific hypothesis of this project is that the new products have similar efficacy in controlling initial bleeding and preventing additional bleeding. This should consequently improve survival.

\section{MATERIALS AND METHODS}

\section{Hemostatic Products}

The products tested were Combat Gauze (CBG) and TraumaStat (TMS). CBG is a tightly rolled 4-yard long, 3-in. wide gauze. The fabric material (non-woven rayon/polyester) is coated with kaolin, an aluminum silicate very potent coagulation initiator that acts as a surface activator [14]. TMS is presented as a 4 -in. $\times 16$-in. and $1 / 16$-in. thick compressed non-woven mesh structure composed of uniquely porous, densely-filled fibers made of chitosan (from shellfish). The chitosanbased pad has both hemostatic and absorbent properties. The manufacturer indicated that TMS contains multiple hemostatic agents mixed with fibers of high surface area for rapid interaction with the clotting components of blood [15]. These two products (weight $\sim 16 \mathrm{~g}$ ) cover similar area in a groin injury. Standard dressing (SD; $\mathrm{H}$ and $\mathrm{H}$ compressed gauze; $\mathrm{H}$ and $\mathrm{H}$ Associates, Bena, VA) was used on top of the test dressings. All products were kindly provided by the manufacturers and used per manufacturers' instruction.

\section{Animal Model}

The experiments reported herein were conducted according to the principles set forth in the Guide for the Care and Use of Laboratory Animals, Institute of Laboratory Animals Resources, National Research Council, National Academy Press, 1996. The study was approved by the Naval Medical Research Center/Walter Reed Army Institute of Research Institutional Animal Care and Use Committee and Uniformed Services University of Health Science. All procedures were performed in accordance to the Animal Welfare Act and in an animal facility approved by the Association for Assessment and Accreditation for Laboratory Animal Care International (AAALAC).

Yorkshire swine, weight range 25-35 kg, (Animal Biotech Industry (Danboro, PA) were refrained from food the night before the experiment but had free access to water. Estimated blood volume (EBV) was calculated as: animal weight $(\mathrm{kg}) \times 65 \mathrm{~mL} / \mathrm{kg}$. Experiments with these models and methods have been previously thoroughly described [1,2]. Briefly, the animals were intubated after anesthesia induction (ketamine $\mathrm{HCl}(30 \mathrm{mg} / \mathrm{kg}$, i.m.) and inhalation of isoflurane $3 \%-4 \%$. The animals were allowed to breathe spontaneously or were mechanically ventilated (Narkomed Ventilator; North American Dräger, Telford, $\mathrm{PA}$ ) if end tidal $\mathrm{CO}_{2}$ and respiration rate fell outside normal values. An 18-20 G angiocatheter and a 7F Introflex introducer were placed in the right carotid artery and the right external jugular vein, respectively, to acquire blood pressure and withdraw blood samples. All catheters were maintained patent $(0.9 \%$ saline solution at $5 \mathrm{~mL} / \mathrm{h}$ ). A temperature probe (Thermometer/Model BAT12; Physitemp, Clifton, NJ) was placed in the groin cavity to monitor change in temperature when the bleeding was started [7]. Vital signs were continuously recorded and rectal temperature was monitored and maintained between $37-39^{\circ} \mathrm{C}$ using a Bair Hugger device (model 505; Augustine Medical, Eden Prairie, MN). A splenectomy was not performed on these animals in contrast to other models [9].

\section{Transection (CBG and TMS, $n=6$ )}

The skin at the right inguinal area of the thigh was incised approximately $10 \mathrm{~cm}$ long and parallel to the groin area. Femoral vessels were accessed by blunt dissection, avoiding vessel constriction or spasm. After recording of baseline parameters, the injury was made by transecting the femoral blood vessels (and the adjacent muscles, partially) to produce an uncontrolled arterial and venous hemorrhage (time 0).

\section{Puncture (CBG and TMS, $n=8$ )}

CBG and TMS were evaluated in this model. The skin of the inguinal area of the right thigh was incised longitudinal to the groin approximately $12 \mathrm{~cm}$ to expose the femoral vasculature. The femoral artery was invasively isolated on $4 \mathrm{~cm}$ on the distal side of the groin. Lidocaine (1\%-2\%) was spread on the artery to maximize dilation of the vessel. Any proximal side branches from the artery were ligated to avoid redirection of blood to the main circulation. Bulldog clamps were placed on the artery and a $4 \mathrm{~mm}$ hole was perforated by an aortic vascular punch (APU440; Medtronic, Minneapolis, MN). Following this injury, the clamps were removed to produce a rapid and immediate high pressure arterial uncontrolled hemorrhage (time 0 ). 
TABLE 1

Baseline, Pretreatment, and Pre-resuscitation Parameters Among the Two Injury Groups

\begin{tabular}{|c|c|c|c|c|c|}
\hline \multirow[b]{2}{*}{ Parameter $($ mean $\pm \mathrm{SD})$} & \multicolumn{2}{|c|}{ Transection } & \multicolumn{3}{|c|}{ Puncture } \\
\hline & CBG & TMS & CBG & TMS & $P$ \\
\hline Weight (kg) & $29.1 \pm 5.2$ & $32.6 \pm 5.4$ & $28.3 \pm 1.1$ & $28.2 \pm 1.9$ & 0.09 \\
\hline Rectal temperature $\left({ }^{\circ} \mathrm{C}\right)$ & $37.0 \pm 0.6$ & $37.7 \pm 0.8$ & $38.0 \pm 0.5$ & $38.2 \pm 0.6$ & 0.06 \\
\hline Initial MAP $(\mathrm{mmHg})$ & $57.5 \pm 18.7$ & $65.2 \pm 21.2$ & $60.0 \pm 6.2$ & $63.6 \pm 3.8$ & 0.32 \\
\hline Pretreatment MAP (mmHg) & $33.2 \pm 14.5$ & $40.8 \pm 24.9$ & $34.4 \pm 5.3$ & $38.6 \pm 10.5$ & 0.72 \\
\hline Pretreatment blood loss (\% EBV) & $25.5 \pm 7.3$ & $23.1 \pm 11.1$ & $18.3 \pm 3.0^{*}$ & $15.4 \pm 3.9^{*}$ & $<0.05$ \\
\hline Rate of loss during injury (mL/ min) & $557 \pm 150$ & $650 \pm 240$ & $417 \pm 87^{*}$ & $340 \pm 103^{*}$ & $<0.05$ \\
\hline MAP before resuscitation ( $\mathrm{mmHg}$ ) & $52.2 \pm 17.4$ & $62.5 \pm 20.8$ & $36.9 \pm 11.7^{*}$ & $32.9 \pm 10.1^{*}$ & $<0.05$ \\
\hline
\end{tabular}

Results are expressed as mean \pm standard deviation.

$\mathrm{MAP}=$ mean arterial pressure; $\mathrm{EBV}=$ estimated blood volume; $\mathrm{CBG}=$ combat gauze $; \mathrm{TMS}=$ TraumaStat.

${ }^{*} P<0.05$ between transection and puncture models for CBG and TMS.

\section{Treatment, Monitoring, and Data Acquisition}

Blood was aspirated from the wound cavity and collected in a sealed container (2L MediVac; Cardinal Health, Dublin, $\mathrm{OH}$ ) and continuously weighed on a top-loading scale (PS 5100; Mettler, Columbus, $\mathrm{OH})$ [volume blood loss $(\mathrm{mL})=$ weight $(\mathrm{g}) / 1.056(\mathrm{~g} / \mathrm{mL})]$. Blood loss was divided into pretreatment and post-treatment hemorrhage. After free bleeding for $2 \mathrm{~min}$ (transection model) or $45 \mathrm{~s}$ (puncture model) (pre-treatment blood loss), the animals were randomly assigned to a treatment group in each respective model by a blinded assistant. The dressing was unfolded and applied one fold at a time with pressure to the wound. Standard dressing was placed over the test dressing, and the wound remained covered for the remainder of the experiment $(3 \mathrm{~h})$. A constant pressure of $\sim 200 \mathrm{mmHg}$ (HM 28 pressure monitor; Dwyer, Michigan City, IN) was applied for 5 min following injury [7, 8, 11]. Then the pressure was released and the wound was closed by clamping the two skin flaps, leaving a residual pressure of approximately 35 $\mathrm{mmHg}$. At $15 \mathrm{~min}$, the animals received resuscitation fluid (fixed volume of isotonic colloidal fluids ( $500 \mathrm{~mL}$ of Hextend; Biotime, Alameda, CA) through the jugular catheter over $30 \mathrm{~min}(\sim 15 \mathrm{~mL} / \mathrm{kg})$ (Masterflex pump; Cole Parmer, Vernon Hills, IL). Animals were euthanized after $180 \mathrm{~min}$ with Euthasol $(0.3 \mathrm{~mL} / \mathrm{kg}$, i.v. $)$.

Recurrence of bleeding (rebleeding) was defined as blood oozing from the dressings (post-treatment blood loss) and requiring aspiration as opposed to no bleeding or bleeding controlled by the dressing (i.e., blood, if any, was retained in the dressing and there was no need for aspiration). Each product was evaluated for recurrence of bleeding following release of the 5 min manual compression and following MAP restoration to $40 \mathrm{mmHg}$. Shed blood contained in test and standard dressings was weighed at the end of the experiment.

Rectal and wound temperatures (at the blood and dressing interface) were continuously recorded using temperature monitors (BAT 12; PhysiTemp, Clifton, NJ). Vital signs (Hewlett Packard, Palo Alto, CA) and pulse oximetry (Transpac IV; Hospira, Lake Forest, Il) were continuously recorded. Blood pressure (MAP, diastolic, systolic), heart rate (HR), $\mathrm{ETCO}_{2}$, isoflurane level, respiration rate, and oxygen saturation were continuously measured. Blood samples $(10 \mathrm{~mL})$ were withdrawn through the arterial line at time $0,5,15$, 45, 60, 120, and $180 \mathrm{~min}$. Blood count (CBC with differential) was measured on a Pentra 60C + cell counter (ABX Diagnostics, Irvine, $\mathrm{CA})$ as standard animal monitoring.

\section{In Vitro Measurements}

For each of the test dressings (CBG, TMS), blood retention ability (absorption) was assessed by weight after pouring $20 \mathrm{~mL}$ of blood on top of $20 \mathrm{mg}$ of product. Clot formation was assessed in test tube with $2 \mathrm{~mL}$ blood recalcified with $\mathrm{CaCl}_{2}$ in which $1 \mathrm{mg}$ of the product was added. Clotting time was measured by tilting the tube $45^{\circ}$ every $30 \mathrm{~s}$ until firm clotting was detected [6]. These tests were performed in triplicate.

\section{Statistical Analysis}

Evaluation of eight animals per group has been sufficient in our studies with these injury models $[1,2,8]$ to distinguish bleeding control and high mortality rates in nontreated groups (100\% mortality [8]) or standard of care (compressed gauze) groups ( $>75 \%$ mortality [8]). Evaluation of CBG and TMS immediately followed a series of similar dressing comparisons $[1,2]$ and therefore no nontreatment or standard of care group was repeated. Power analysis on blood loss (continuous variable) could differentiate between dressings. Furthermore, in the case four animals consecutively survived the $3 \mathrm{~h}$ experiment with both dressings in a model, two additional animals were tested and the total number of animals was re-evaluated. The group number remained at six if $100 \%$ survival and similar outcomes to the rest of the group were achieved, as was the case for the transection model here. This procedure was intended to reduce the use of animals in this experiment to only those needed for research objectives. Each injury model was treated independently and only the dressing within each model was randomly assigned. ANOVA, Kaplan Meier, and Fisher Exact tests were performed (Statistix, Tallahassee, FL; SAS Institute, Inc., Cary, NC). Data are presented as mean \pm standard deviation and $P<0.05$ was considered significant.

\section{RESULTS}

A total of 12 pigs were used in the transection model, $n=6$ for CBG and for TMS. A total of 17 pigs were used in the puncture model, $n=8$ for CBG and for TMS, and 1 pig died within 15 min due to severe initial injury (hemorrhagic shock) and was not included in the data analysis. There was no difference in baseline levels of weight and vital signs (temperature, MAP, or HR) between treatment groups (CBG or TMS) or between experimental injury groups (transection versus puncture). Baseline parameters are listed in Table 1. All animals had normal hematology, coagulation, and serum chemistry (not shown). Immediately after injury, there was no difference in pretreatment MAP between treatment groups or between the respective experimental 
TABLE 2

Rate and Time of Survival

\begin{tabular}{llcc}
\hline & \multicolumn{3}{c}{ Survival time } \\
\cline { 2 - 4 } & \multicolumn{1}{c}{ Combat gauze } & TraumaStat & $P$ \\
\hline Transection & $180 \min (6 / 6)$ & $180 \min (6 / 6)$ & $=1$ \\
Puncture & $174 \pm 16 \min (7 / 8)$ & $153 \pm 55 \min (4 / 8)$ & $>0.2$ \\
\hline
\end{tabular}

Average time is expressed as mean \pm standard deviation.

None of these values reached statistical significance.

Ratio of surviving animals is included in parenthesis.

injury groups transection $(37.0 \pm 14.5 \mathrm{mmHg}$, at $2 \mathrm{~min})$ versus puncture $(35.2 \pm 8.0 \mathrm{mmHg}$ at $45 \mathrm{~s})$. However, blood loss was greater in the transection model than in the puncture model $(24 \% \pm 8 \% \mathrm{EBV} 17 \% \pm 4 \%$ EBV, respectively; $P<0.05$ ).

Survival rate in the transection model was $100 \%$ for both CBG and TMS dressings (Table 2). In the puncture model, survival rates were less than $100 \%$ [88\% for CBG, 50\% for TMS (no statistical difference)]. Similarly, there was no statistically significant difference between groups in mean survival time (174 min for CBG and 153 min for TMS). This dynamic is illustrated in a Kaplan Meier plot (Fig. 1) indicating that animals died after $90 \mathrm{~min}$ for TMS and after $120 \mathrm{~min}$ for CBG (median time) in the puncture model.

Aspirated blood was significantly less in the transection model than in the puncture model. Total posttreatment blood, as a measure of control of bleeding from the dressing, showed a lower mean with CBG treatment than with TMS treatment in the puncture model $(28.6 \% \pm 25.2 \%$ versus $43.3 \% \pm 19.4 \% \mathrm{EBV}$, respectively) but this mean difference was not statistically significant. Amount of blood in the standard dressing (applied on top of the test dressing) was similar in CBG and TMS treatment groups but less in the transection model than in the puncture model.

Rebleeding was evident at two specified events: release of manual compression and restoration of

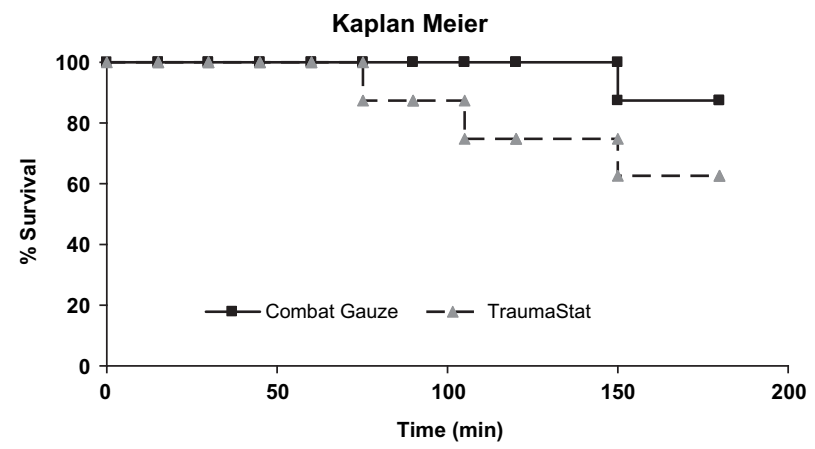

FIG. 1. Survival across all dressings: Kaplan Meier plot in puncture model.
MAP after injury ( $40 \mathrm{mmHg}$, which occurred between time 20 and 45, during fluid resuscitation; Table 3). In the transection model, rebleeding incidence was $0 \%$ for CBG and $17 \%$ for TMS without significant reduction of MAP post-treatment, allowing the animals to survive the $3 \mathrm{~h}$ with a final MAP above $60 \mathrm{mmHg}$. In the puncture model, rebleeding occurred frequently at compression release (Table 3 ), causing a significant reduction in MAP to an average of $25.0 \pm 8.0 \mathrm{mmHg}$ (lowest MAP, Table 3) compared with pre-treatment MAP (average $35.2 \pm 8.0 \mathrm{mmHg}, P<0.01$, ANOVA). Thereafter, MAP increased due to compensation regardless of dressing (MAP before resuscitation, Table 1). After resuscitation and MAP increasing steadily to a maximum, similar for CBG, and TMS, treatments (59 $\pm 17,54 \pm$ $13 \mathrm{mmHg}$, respectively, $P=0.3$, ANOVA), incidence of rebleeding occurred similarly with both dressings but there was less blood loss with CBG $(P<0.05)$. At the end of the experiment, MAP reached approximately $44 \mathrm{mmHg}$.

In this circumstance, the animals surviving the puncture injury ended the experiment with a lower MAP $(P<0.01)$ than the transection injury.

\section{Wound Temperature}

Increase of temperature has been a concern with zeolite [8] in the past but no such temperature increase was observed after application of the two test dressings in this study. The temperature recorded at the interface of the products and the inguinal muscle was no different than rectal temperature for all animals across time, groups, and models $\left(37.5 \pm 1.4^{\circ} \mathrm{C}\right)$.

\section{Additional Characteristics of Test Hemostatic Dressings}

Additional observations of dressing properties made during in vitro experiments are described in Table 4. CBG has greater absorption than TMS $(P<0.05)$. CBG has a greater ability to promote clotting than TMS $(P<0.05)$. During in vivo experiments, each dressing was subjectively evaluated at each treatment for ease of application and difficulty of removal, using a 3-level rating scale. These actions, application, and removal presented no difficulty for either test dressing.

\section{DISCUSSION}

Combat Gauze and TraumaStat are two fabric-based hemostatic dressings newly released at the time of this experiment that showed potential immediate application for battlefield deployment. Both are lightweight and are suitable for long-term storage under a wide temperature range. We tested them in two injury models: a full femoral vessel transection and a $4 \mathrm{~mm}$ 
TABLE 3

Post-Treatment Events and Parameters Observed in Transection and Puncture Models

\begin{tabular}{|c|c|c|c|c|c|c|c|c|}
\hline \multirow[b]{2}{*}{ Dressings } & \multicolumn{2}{|c|}{ Incidence of rebleeding } & \multicolumn{4}{|c|}{ MAP after dressing application $(\mathrm{mmHg})$} & \multicolumn{2}{|c|}{ Blood loss during rebleeding (\% EBV) } \\
\hline & at CR & at MAP R & Lowest MAP & Highest MAP & $\operatorname{Time}^{\dagger}(\min )$ & $\mathrm{MAP}$ at $3 \mathrm{~h}$ & at CR & At MAP R \\
\hline CBG & $0 \%$ & $0 \%$ & $33 \pm 15$ & $77 \pm 21$ & $58 \pm 19$ & $68 \pm 13$ & 0 & 0 \\
\hline \multirow[t]{2}{*}{ TMS } & $17 \%$ & $17 \%$ & $41 \pm 25$ & $88 \pm 13$ & $59 \pm 26$ & $72 \pm 10$ & 1.3 & 2.8 \\
\hline & \multicolumn{8}{|c|}{ Puncture } \\
\hline
\end{tabular}

Incidence of rebleeding, MAP, and blood loss in the transection and puncture models.

Incidence of rebleeding is indicated after compression (CR) and after restoration of MAP (MAP R, $\geq 40 \mathrm{~mm} \mathrm{Hg}$ ) and is calculated as \% of animals that had blood aspirated from the wound.

Results are expressed as mean \pm standard deviation.

MAP = mean arterial pressure, CBG; Combat Gauze, TMS; TraumaStat.

Pre-T = pretreatment blood loss; Asp = aspirated blood loss; Post-R = post-resuscitation blood loss.

* $P<0.05$

${ }^{\dagger}$ Time at which MAP was the highest. MAP is subdivided as: lowest MAP after compression and before fluid resuscitation, peak MAP (highest MAP maintained after dressing application and time (min) it occurred), MAP at end point.

arterial punch $[1,2]$. These models provide challenges to the hemostatic dressing to control bleeding in two ways: (1) recurrence of bleeding immediately after release of the manual compression, and (2) recurrence of bleeding after MAP has rebounded.

In the transection model, where standard gauze yielded less than $20 \%$ survival in a previous study with this injury model, both dressings demonstrated $100 \%$ survival, and were comparable to the top ranked dressings in the previous evaluation [1]. In the puncture model, CBG yielded comparable survival to a precursor product, X-Sponge $(87.5 \%$ and $75 \%$, respectively), which was ranked as a superior dressing in a previous evaluation [2]. In contrast, TMS ranked lower than a product of similar chitosan composition, Celox (50\% and $88 \%$, respectively) [2].

CBG showed a similar incidence of rebleeding in the puncture model as its precursor X-Sponge, (50\% and $60 \%$, respectively), similar volume of blood loss $(20 \%$ EBV aspirated blood), and similar survival (75\% and

\section{TABLE 4}

Characteristics of Hemostatic Dressings

\begin{tabular}{lcc}
\hline Hemostatic dressings & $\begin{array}{c}\text { Absorp factor } \\
(\mathrm{g} / \mathrm{g})\end{array}$ & $\begin{array}{c}\text { In vitro clotting } \\
\text { time }^{\ddagger}(\mathrm{s})\end{array}$ \\
\hline Gauze dressings & & \\
Combat gauze $n=6$ & $7.1 \pm 0.1$ & $105 \pm 16^{*}$ \\
TraumaStat $n=6$ & $5.7 \pm 0.1^{*}$ & $190 \pm 16$ \\
$*$ & $P<0.05$ & $P<0.01$ \\
\hline
\end{tabular}

In vitro properties of dressing material tested in the laboratory.

"Level of significance determined with ANOVA.

${ }^{\dagger}$ Absorption factor: weight of blood absorbed/weight of dressing $(\mathrm{g} / \mathrm{g})$.

${ }^{\ddagger}$ clotting time (s) determined with $2 \mathrm{~mL}$ blood at $37^{\circ} \mathrm{C}$.
87.5\%). Rebleeding with TMS was associated with higher aspirated blood loss than Celox; products made of the same base ingredient (chitosan). There was a trend of higher incidence of rebleeding after MAP restoration with TMS compared with CBG. The performance of CBG in supporting bleeding control resulted in a slightly but not statistically higher survival rate than for TMS in the puncture model. Although not statistically significant, this survival rate, taken together with the lower incidence of rebleeding and lower volume of blood loss, was consistent with lower TMS performance.

Mechanism of action of these dressings may be associated with their absorption and clotting abilities, which were seen as important characteristics with previous testing of other hemostatic dressings [1]. The lower ability of TMS to control bleeding with restoration of MAP may be due to the matrix support permitting rapid initial blood absorption but less ability to control secondary bleeding. These differences may be due to the space/volume occupied by the products in the wound. When filled with blood, CBG may have achieved a better contact with the wound surface and consequently exerted a more homogeneous wound compression, preventing further blood flow. We have noted that the fabric used in CBG ensured good initial contact and absorption that is important in the initial hemostasis (data not shown). In vitro data indicate that this product, impregnated with hemostatic agent (kaolin), provides additional properties that yield good hemostatic control in vivo, as seen in the maintenance of higher MAP. The physical support (texture) of fabric-like dressings might also play a large role in the dressing properties. Furthermore, in supporting 
observations from a separate study of hemostatic dressings, Kheirabadi et al. reported that CBG was more effective than TMS in bleeding control and provided better bleeding control than the standard of care dressing (compressed gauze) (personal communication, [16]. Interestingly, they outlined the importance of the nature of the support material in gauze dressing property.

Incidence of rebleeding during resuscitation indicates that there are higher risks of rebleeding for any dressings while under high pressure hemorrhage. This was clearly observed in the puncture model, the more challenging model in this respect. It was notable that in the puncture model, after injury, MAP was reduced to the same level as in the transection by less blood loss. This is likely caused by a shorter bleeding time at high rate. However, due to higher rebleeding after compression release in the puncture model, more blood loss occurred and caused MAP to decrease further before fluid resuscitation. In the transection model, little blood loss occurred after application of either dressing. The reason for this difference between models is not clear. It could be attributed to the retraction of the vessels and constriction of the artery after full transection, and the high risk of bleeding in the puncture injury on a fully dilated vessel.

In consideration of some limitations, this study was designed to evaluate two dressings that may have clinical potential for bleeding control. Additional comparisons between dressings could have been made, had a larger number of animals been used. The fluid resuscitation limit of $500 \mathrm{~mL}$ is a second limitation and might have left the animal hypotensive; however, this study was designed for military application where hypotensive resuscitation is recommended to simulate austere situation [17]. Administration of this smaller volume of colloid infusion compared with a greater volume of crystalloid might have affected the study's outcomes such as additional rebleeding and lower survival. In this regard, surviving animals could have been subdivided into groups that had stable MAP to the end of experiment or that had MAP decrease towards the end of the experiment. For the animals that did not die within the experimental time but had decreasing MAP, loss of hemodynamics could be attributed to substantial blood loss occurring either before or after the resuscitation regimen. This suggests that if experimental time had been prolonged, the survival rate might be different. Ultimately, these animal models are research tools only and do not convey to direct translation with a human scenario on a battlefield. However, swine is an appropriate cardiovascular model with MAP and response to hemorrhagic shock and resuscitation fluid approaching that in human.

\section{CONGLUSION}

Overall, CBG and TMS dressings were equally efficacious for bleeding control after severe hemorrhage in the transection injury model. Departure between these dressings becomes distinguishable in a more challenging puncture injury model, conceding a slight advantage to CBG. Both dressings have clear advantages for hemostasis over standard of care dressing. Importantly, these new dressings retain rather than sacrifice the fabric-like properties of standard of care dressings already familiar to care providers.

\section{ACKNOWLEDGMENTS}

The authors thank Drs. T. Tomori, A. Scultetus, A. Haque, M. Hammett, B. Kim, and N. Carballo for surgical and technical assistance, and J. Norris for editorial support. The authors are grateful to ZMedica, Wallingford, CT and OreMedix for providing the test materials. Research effort was supported by MARCORSYSCOM 206623 M.162 M.A0607.

Disclosure of commercial interest: None; no author has any commercial or financial interests. There are no transfers of funds between NMRC and product manufacturers in either of these agreements.

\section{REFERENCES}

1. Arnaud F, Parreño-Sacdalan D, Tomori T, et al. Comparison of ten hemostatic dressings in a groin transection model in swine. $\mathrm{J}$ Trauma 2009. in press.

2. Arnaud F, Teranishi K, Tomori T, et al. Comparison of ten hemostatic dressings in a groin puncture model in swine. J Vasc Surg 2009;50:632.

3. Kortbeek JB, Al Turki SA, Ali J, et al. Advanced trauma life support, 8th edition, the evidence for change. J Trauma 2008;64:1638.

4. Hoyt RW, Reifman J, Coster TS, et al. Combat medical informatics: Present and future. Proc AMIA Symp 2002;335.

5. Soreide K. Three decades (1978-2008) of advanced trauma life support (ATLS) practice revised and evidence revisited. Scand J Trauma Resusc Emerg Med 2008;16:19.

6. Arnaud F, Hammett M, Fasipe D, et al. Evaluation of in vitro properties of hemostatic agents. Proceedings of the ATACCC, St Petersburg, FL; August, 2007; [Abstract].

7. Alam HB, Chen Z, Jaskille A, et al. Application of a zeolite hemostatic agent achieves $100 \%$ survival in a lethal model of complex groin injury in swine. J Trauma 2004;56:974.

8. Arnaud F, Tomori T, Saito R, et al. Comparative efficacy of granular and bagged formulations of the hemostatic agent QuikClot. J Trauma 2007;63:775.

9. Kheirabadi BS, Acheson EM, Deguzman R, et al. Hemostatic efficacy of two advanced dressings in an aortic hemorrhage model in swine. J Trauma 2005;59:25.

10. Carraway JW, Kent D, Young K, et al. Comparison of a new mineral based hemostatic agent to a commercially available granular zeolite agent for hemostasis in a swine model of lethal extremity arterial hemorrhage. Resuscitation 2008;78:230.

11. Kozen BG, Kircher SJ, Henao J, et al. An alternative hemostatic dressing: Comparison of CELOX, HemCon, and QuikClot. Acad Emerg Med 2008;15:74.

12. Wedmore I, McManus JG, Pusateri AE. The chitosan-based hemostatic dressing: Experience in current combat operations. J Trauma 2006;60:655.

13. Kheirabadi BS, Sherer MJ, Estep JS, et al. Comparison of new hemostatic granules/powders with currently deployed hemostatic products in a lethal model of extremity arterial hemorrhage in swine. J Trauma 2009;66:316. 
14. http://www.z-medica.com/products/quikclot-combat-gauze.asp.

15. http://www.oremedix.com/news/default.asp?theArticle=1.

16. Kheirabadi BS, Sherer MJ, Estep JS, et al. Comparison of new hemostatic dressings with currently deployed HemCon bandages in a model of extremity arterial hemorrhage in swine.Proceedings of the EAST meeting, Lake Buena Vista, FL; Jan, 2009; [Abstract].

17. Champion HR. Combat fluid resuscitation: Introduction and overview of conferences. J Trauma 2003;54(5 Suppl):S7. 